\title{
NICE must do better at summarising and communicating evidence on statins
}

\author{
Ben M Goldacre research fellow in epidemiology \\ London School of Hygiene and Tropical Medicine, London WC1E 7HT, UK
}

Rabar and colleagues' summary of the National Institute for Health and Care Excellence (NICE) guideline on statins omits the key information clinicians need: the number needed to treat (NNT) with statins in different risk groups, at different levels of treatment intensity. ${ }^{1}$ This is not The BMJ's shortcoming. NICE advises doctors to discuss this information with their patients, but it is missing from the NICE guideline itself.

The most attentive reader might find-in row 10 of 32 , in table 43 , on page 143 - that for every 1000 people without cardiovascular disease taking a statin, overall there would be seven fewer non-fatal myocardial infarctions. To establish the time period over which this figure applies, or whether it relates to the important new $10 \% 10$ year risk population, requires downloading and reading Appendix C, a separate document. Table 60 summarises data from a 2013 Cochrane review, which (arguably) relates to a 15\% 10 year risk population, and reports an NNT of 88 for "total CHD events" over five years. Attentive readers could deduce that these events must be non-fatal, or perhaps mixed fatal and non-fatal, because the NNT for all cause mortality in the same table is higher.

NICE advises doctors to give clear information on the benefits of statins. This implies that, after scrutinising a 302 page NICE report to find the numbers above, GPs should then do a literature search, read and appraise the trial data, synthesise it (at different risk strata and treatment intensities), then use the appropriate formulas to create NNT for easy interpretation by patients. Clinicians might expect NICE—a well resourced national body with extensive technical expertise - to have done this for them. Clear summaries of information on benefit and risk are the bedrock of informed patient choice. They should be our highest priority, ${ }^{2}$ not a poor second cousin. A simple table in NICE's own summaries-giving NNTs we know, and highlighting those we don't —might be a good place to start. I hope others will have more suggestions.

Competing interests: I receive income from writing and speaking to lay and professional audiences on problems in science, including poor communication of risk and badly designed trials.

Full response at: www.bmj.com/content/349/bmj.g4356/rr/761330.

1 Rabar S, Harker M, O'Flynn N, Wierzbicki AS; on behalf of the Guideline Development Group. Lipid modification and cardiovascular risk assessment for the primary and secondary prevention of cardiovascular disease: summary of updated NICE guidance. BMJ 2014;349:g4356. (17 July.)

2 Goldacre B, Smeeth L. Mass treatment with statins. BMJ 2014;349:g4745. (23 July.)

Cite this as: BMJ 2014;349:95081

๑ BMJ Publishing Group Ltd 2014 